\title{
Dynamics of Adatom Motion under the Influence of Mutual Interactions: $\mathrm{O} / \mathrm{Ru}(\mathbf{0 0 0 1})$
}

\author{
S. Renisch,* R. Schuster, J. Wintterlin, and G. Ertl \\ Fritz-Haber-Institut der Max-Planck-Gesellschaft, Faradayweg 4-6, D-14195 Berlin, Germany
} (Received 1 September 1998)

\begin{abstract}
The diffusive motion of $\mathrm{O}$ atoms adsorbed on $\mathrm{Ru}(0001)$ was observed on the atomic scale by scanning tunneling microscopy with temporal resolution of $\approx 10 \mathrm{~ms}$ at room temperature. From statistical analysis of the changes of the atom configurations in sequences of more than 1000 images, mean residence times of oxygen atoms in the neighborhood of other oxygen atoms were extracted. The residence times vary by more than 1 order of magnitude over distances of three lattice constants, indicating the necessity of including the lateral interaction in modeling collective phenomena such as surface diffusion or reactions. [S0031-9007(99)09081-X]
\end{abstract}

PACS numbers: 68.35.Fx, 82.65.Dp

The motion of adsorbed particles governs many important surface processes, such as catalytic reactions, crystal growth, or epitaxy. All of these processes involve nonzero coverages, and the particles are known to interact considerably with each other (as evident, e.g., from the observation of island formation or the variation of the adsorption energy with coverage). Thus, it has to be concluded that also their motions are not independent of each other. Although this problem (which is reflected, for example, by the coverage dependence of the macroscopic diffusion coefficient) has been recognized for many years [1], direct microscopic studies on the effects of mutual interactions on the dynamics of adatom motion are still lacking except for a few special cases.

Interactions between adsorbed metal atoms have been studied extensively by field ion microscopy (FIM) [2,3]. These (static) experiments measured effects on the potential energy minima at the adsorption sites that determine the equilibrium distribution of the atoms, and revealed interesting results about long range effects [4]. The motion of the particles is, however, governed also by the potential maxima (in principle by the complete potential caused by the interaction with the substrate and neighboring adsorbates). Conclusions about the dynamics were so far focused on the question of "long jumps" [3]. Recently, the motion of particles could be followed by scanning tunneling microscopy (STM), providing data on the motion of individual particles and also on long or double jumps [5]. Results on mutual effects of neighboring particles on the dynamics exist only for the special case of motion in one dimension [6,7], whereas the general 2D case was not treated. Since here all hopping events into the various directions can be affected by the presence of another adparticle, the evaluation of statistically significant numbers requires enormous data sets that have not been available so far.

In this Letter, we present results for the 2D motion of adsorbed particles under the influence of their neighbors for the system $\mathrm{O} / \mathrm{Ru}(0001)$. Quantitative data of this kind could, so far, not be obtained by STM with conventional scanning speed because the residence time of an adsorbate particle on a lattice site has to be of the order of several seconds to minutes to allow its observation. With a special fast STM system, we were able to study atoms with much shorter residence times $(\$ 10 \mathrm{~ms})$ and to record much larger data sets within reasonable times. By a detailed statistical analysis of the hopping events, we could quantify variations of the residence time under the influence of interactions. Near a second oxygen atom residence times are affected over distances up to three lattice constants and vary by up to 1 order of magnitude. These effects are, hence, of importance already at very low coverages.

The experiments were performed in a UHV chamber with a base pressure of $5 \times 10^{-11}$ mbar equipped with the fast STM and facilities for sample preparation and characterization [8]. STM image sequences were recorded at room temperature at frequencies of 8 to $20 \mathrm{images} / \mathrm{s}$. Each sequence typically consists of several hundred to some thousand images. As an example, Fig. 1 shows two successive images from a sequence of $\approx 2200$ images, taken at a frequency of $18 \mathrm{~s}^{-1}$ on a surface with an oxygen coverage of 0.014 monolayers (ML). The oxygen atoms are imaged as bright dots, as described previously [8].

Although the Ru substrate lattice was not resolved in these data, it could be reconstructed from the positions of
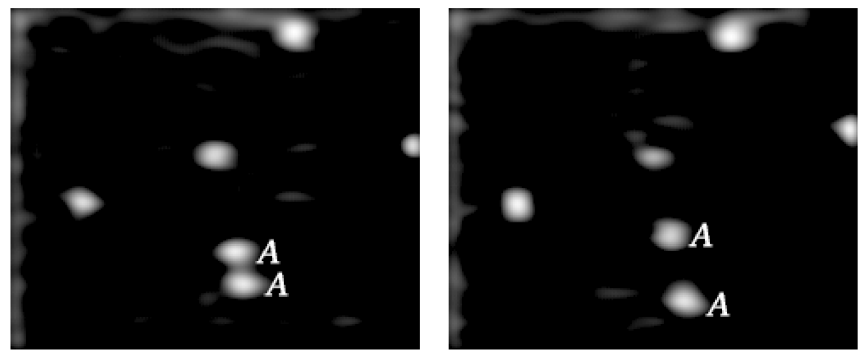

FIG. 1. Two successive tunneling images $(35 \AA \times 55 \AA)$ from a sequence of more than 2200 images $\left(U_{T}=-0.23 \mathrm{~V}\right.$, $I_{T}=10 \mathrm{nA}$ ); the letters A mark the atoms of a dimer with an initial distance of $2 a_{0}$, both of which moved between the images, resulting in a distance of $4 a_{0}$. 
the oxygen atoms. This is justified by the previous observation [8] that all $\mathrm{O}$ atoms occupy the same (hcp) site of the $\mathrm{Ru}(0001)$ surface. With the knowledge of the $(1 \times 1)$ lattice, the displacement vectors of the single atoms between subsequent images were extracted. With this procedure, which will be published in detail elsewhere [9], about $99 \%$ of the atoms could be assigned to unequivocal positions; all remaining events were discarded.

As a reference process the motion of isolated particles was studied. For this purpose experiments with oxygen coverages between $\Theta=0.01$ and 0.02 ML were performed; the average distances between the oxygen atoms are then of the order of 5 to $10 a_{0}, a_{0}=0.271 \mathrm{~nm}$ being the $\mathrm{Ru}$ lattice constant. Figure 2 shows a histogram of the displacements of adatoms between two subsequent STM images, where in both, the initial and the final configuration, the analyzed atoms were more than $3 a_{0}$ away from the nearest-neighboring $\mathrm{O}$ atoms. A total of more than 3300 events was observed in a sequence of 2200 images. The distribution in Fig. 2 does not change when atoms more than $4 a_{0}$ separated from their neighbors were selected. Atoms separated by more than $3 a_{0}$ can therefore be regarded as isolated. Experiments with higher coverages led to the same results, indicating the absence of cumulative long range effects. It is noteworthy that the distribution of the jumps is completely isotropic; i.e., it does not show any correlation with the scanning direction. Careful checks for a possible STM tip effect revealed that it remained negligible as long as the tunneling resistance did not become very low $\left(<10^{6} \Omega\right)$.

Since in Fig. 2 most of the atoms are seen to be displaced by at least one lattice site, the possibility of multiple jumps between two frames has to be taken into account. Assuming that the isolated adparticles perform a random walk, consisting only of jumps between

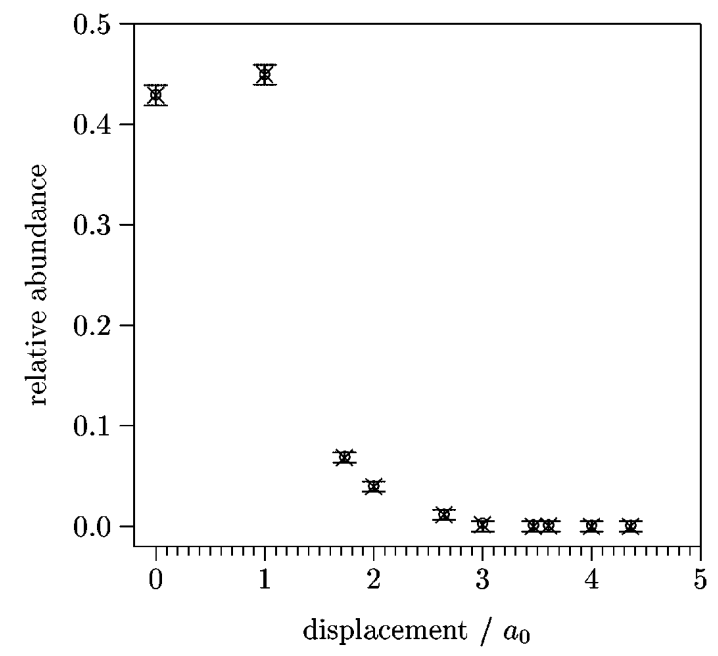

FIG. 2. Jump width distribution for isolated atoms. Experimental values (crosses) are given with error bars representing the square root of the number of observed events; the fit is marked by open circles. nearest-neighbor sites, it is possible to give exact analytic expressions for the probability that a particle is displaced by a given lattice vector after time $t$ [10]. The only adjustable parameter in this probability distribution is the inverse residence time of a particle on a particular site, or hopping frequency $\Gamma$. This distribution is fitted to our experimental data, yielding a hopping frequency of $\Gamma=$ $16.6 \pm 0.9 \mathrm{~s}^{-1}$. The hopping frequency corresponds to a mean residence time of $60 \mathrm{~ms}$, in agreement with estimates from "slow" STM data [8]. The fit matches the data points accurately, indicating the validity of the assumption of statistically independent jumps, and, in particular, the absence of long jumps. If we allow in the fitting procedure also for jumps by $2 a_{0}$, the hopping frequency does not change, but the fitted fraction of long jumps remains well below 1\%. By assuming a prefactor between $10^{10}$ and $10^{13} \mathrm{~s}^{-1}$, a diffusion barrier in the range of $E_{D} \approx 0.55-0.7 \mathrm{eV}$ is estimated.

In order to get information on how the residence time of the oxygen atoms is influenced by neighboring $\mathrm{O}_{a d}$ atoms, the motion of dimers was analyzed. We define these as complexes consisting of two adatoms, each of which is separated from all other adatoms, except its dimer counterpart, by at least $3 a_{0}$. From the set of all dimers that could be identified in the STM images, histograms of the changes of the configurations in subsequent frames were assembled, grouped together according to the sixfold symmetry of the lattice of the adsorption sites. Each of these histograms (one per initial configuration) results from the superpositions of the motions of the two atoms. Similarly to the independent atoms, these can have performed several jumps and can have traveled various different paths between successive frames; moreover, we allow for mutual influences on the jump frequencies, depending on the distances between the dimer atoms at each instant. Hence, in order to obtain values for the residence times in the various configurations, the histograms have to be disentangled into the different processes. This was performed by describing the stochastic processes by their master equations $[10,11]$. If $p_{i, j}(t)$ denotes the probability to find a particular particle on site $(i, j)$, the evolution of $p_{i, j}(t)$ is described as a Markov process by a set of master equations,

$$
\frac{\partial}{\partial t} p_{i, j}(t)=\sum_{i^{\prime}, j^{\prime}} \gamma\left(i j \mid i^{\prime} j^{\prime}\right) p_{i^{\prime}, j^{\prime}}(t)-p_{i, j}(t) \sum_{i^{\prime}, j^{\prime}} \gamma\left(i^{\prime} j^{\prime} \mid i j\right),
$$

where the $\gamma\left(i j \mid i^{\prime} j^{\prime}\right)$ are transition rates from state $(i, j)$ to state $\left(i^{\prime}, j^{\prime}\right)$. Based on our results on the isolated particles, we allow only for nearest-neighbor jumps, i.e., the transition rates $\gamma\left(i j \mid i^{\prime} j^{\prime}\right)$ are nonzero only if $(i, j)$ and $\left(i^{\prime}, j^{\prime}\right)$ are neighboring sites. Without interaction with other particles the diffusion process is isotropic and independent of the specific site. In this case, the transition rate to one of the six neighboring sites is given by $\gamma_{0}=\Gamma / 6$, where $\Gamma$ is the hopping frequency derived 


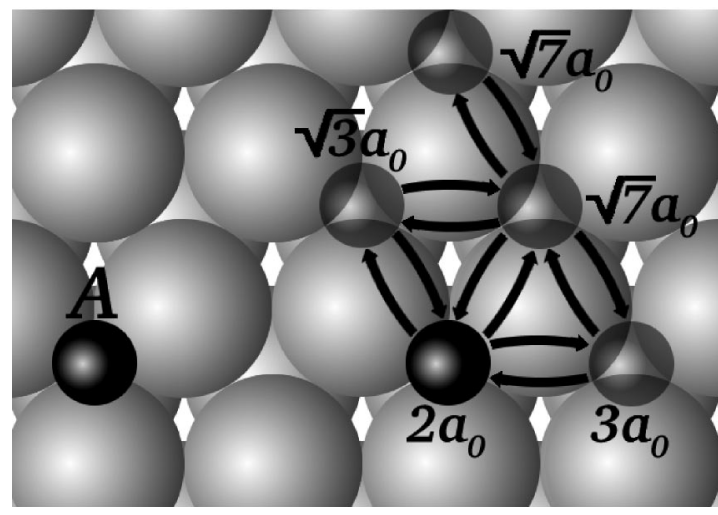

FIG. 3. Model for dimer motion. Paths (indicated by arrows) along which the hopping rates of an atom are affected by the atom A. The nearest-neighbor sites are not occupied under the conditions of our measurements because of strong repulsions. Hopping rates to sites further away are the same as of independent atoms.

above for the isolated particles. Under the influence of another adparticle the hopping rates $\gamma$ are modified. For a quantitative analysis, the probability $\tilde{p}(\vec{\Delta})$ to find a dimer with a relative distance vector $(\tilde{i}, \tilde{j})=\vec{\Delta}$ is introduced for which [by means of Eq. (1)] a new master equation can be derived [9]:

$$
\begin{aligned}
\frac{\partial}{\partial t} \tilde{p}(\vec{\Delta})= & \sum_{\vec{\Delta}^{\prime}=\mathrm{NN}(\vec{\Delta})}\left[w\left(\vec{\Delta}, \vec{\Delta}^{\prime}\right)+w\left(-\vec{\Delta},-\vec{\Delta}^{\prime}\right)\right] \tilde{p}\left(\vec{\Delta}^{\prime}\right) \\
& -\tilde{p}(\vec{\Delta}) \sum_{\vec{\Delta}^{\prime}=\mathrm{NN}(\vec{\Delta})}\left[w\left(-\vec{\Delta}^{\prime},-\vec{\Delta}\right)+w\left(\vec{\Delta}^{\prime}, \vec{\Delta}\right)\right] .
\end{aligned}
$$

$\mathrm{NN}(\vec{\Delta})$ denotes the displacement to nearest-neighbor sites of $\vec{\Delta} . w\left(\vec{\Delta}, \vec{\Delta}^{\prime}\right)$ is the rate at which a particle located at a distance $\vec{\Delta}^{\prime}$ from the other particle jumps to a distance $\vec{\Delta}$. In order to obtain values for the modified hopping rates $w$, Eq. (2) was integrated numerically, resulting in theoretical histograms for the changes of the configurations of dimers with initial separations $\Delta$. These were then fitted to the experimental histograms by variation of the various hopping rates. This analysis was performed for dimers with initial distances of $\sqrt{3}, 2, \sqrt{7}$, and 3 lattice constants (see Fig. 3); Fig. 4 shows two of the four experimental histograms together with the respective fits. Larger distances could be neglected as demonstrated above, dimers with a distance of $1 a_{0}$ were never observed in these experiments. Only at coverages $\Theta>0.25$ structures containing this small separation are formed, which is, however, associated with strong repulsion [12]. For each of the above-mentioned distances, a histogram containing 10 to 15 data points was obtained; the four histograms were then fitted simultaneously by adjusting 10 hopping rates for the various processes.

The total hopping rate out of a site, or the reciprocal residence time on this site, is the sum of the hopping rates to all neighboring sites,

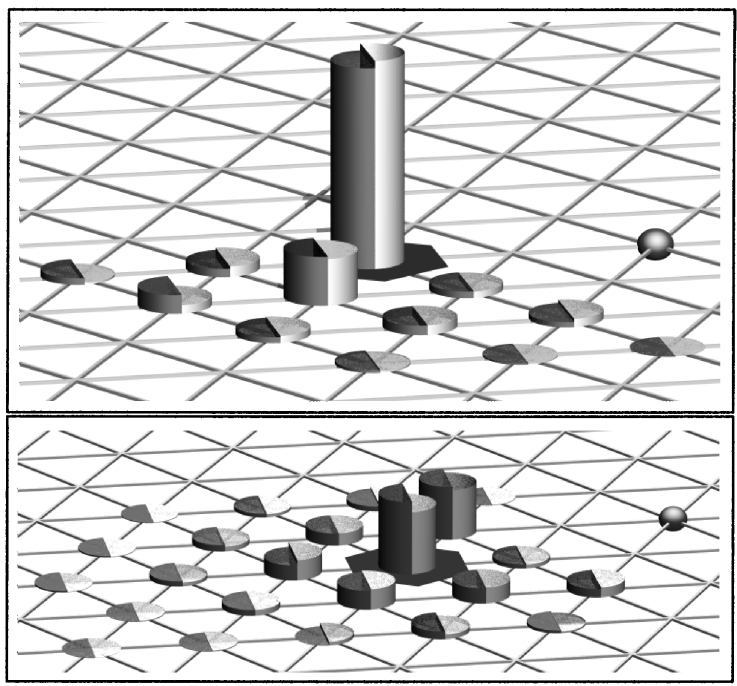

FIG. 4. Histograms for motion of dimers with initial distances of $2 a_{0}$ (upper histogram, 427 events) and $\sqrt{7} a_{0}$ (lower histogram, 334 events); dark bars: experimental values; bright bars: fit; the sphere denotes the place of one atom of the dimer, the shaded hexagon the initial position of the other.

$$
\Gamma_{\vec{\Delta}}=\sum_{\vec{\Delta}^{\prime}=\mathrm{NN}(\vec{\Delta})} \gamma\left(\vec{\Delta}^{\prime} \mid \vec{\Delta}\right) .
$$

Table I summarizes the residence times for the different dimer separations. The errors stem from the fit of the hopping rates and reflect the temporal resolution of our fast STM of about $20 \mathrm{~ms}$. Compared to $\tau_{\infty}=60 \mathrm{~ms}$ for the isolated particles, the residence time at a distance of $2 a_{0}$ is significantly higher, whereas it decreases on the $3 a_{0}$ and $\sqrt{3} a_{0}$ sites. In contrast to recent work that showed collective processes for $\mathrm{CO}$ on $\mathrm{Cu}(110)$ [13], there is no indication for any time correlation between the hopping events of the individual atoms of a dimer. This follows from the observation of the center-of-mass motion of dimers, which was found to be consistent with the assumption of single, noncorrelated jumps.

Finally, the hopping rates were transformed into a pairwise interaction potential between the oxygen atoms. The various hopping rates are given by $\gamma^{\prime}=\nu e^{-E_{D}^{\prime} / k T}$, where $E_{D}^{\prime}$ is the energy barrier in the direction of the jump, and $\nu$ is the frequency factor. Since the diffusion barrier $(\approx 0.55$ to $0.7 \mathrm{eV}$ ) is much larger than the modification by the interactions (few $10 \mathrm{meV}$, see below), the preexponential factors can be assumed to be about equal [14]. For the ratios of the jump rates between two sites, the preexponential factors cancel [15]: $\gamma_{i \rightarrow j} / \gamma_{j \rightarrow i}=e^{-\left(E_{i}-E_{j}\right) / k T}$; the resulting energy differences represent the pairwise

TABLE I. Residence times (in $\mathrm{ms}$ ) at the various dimer separations.

\begin{tabular}{ccccc}
\hline \hline$\tau_{\infty}$ & $\tau_{2}$ & $\tau_{3}$ & $\tau_{\sqrt{7}}$ & $\tau_{\sqrt{3}}$ \\
\hline 60 & 220 & 43 & 66 & 14 \\
\pm 3 & \pm 36 & \pm 6 & \pm 12 & $+18 /-10$ \\
\hline \hline
\end{tabular}




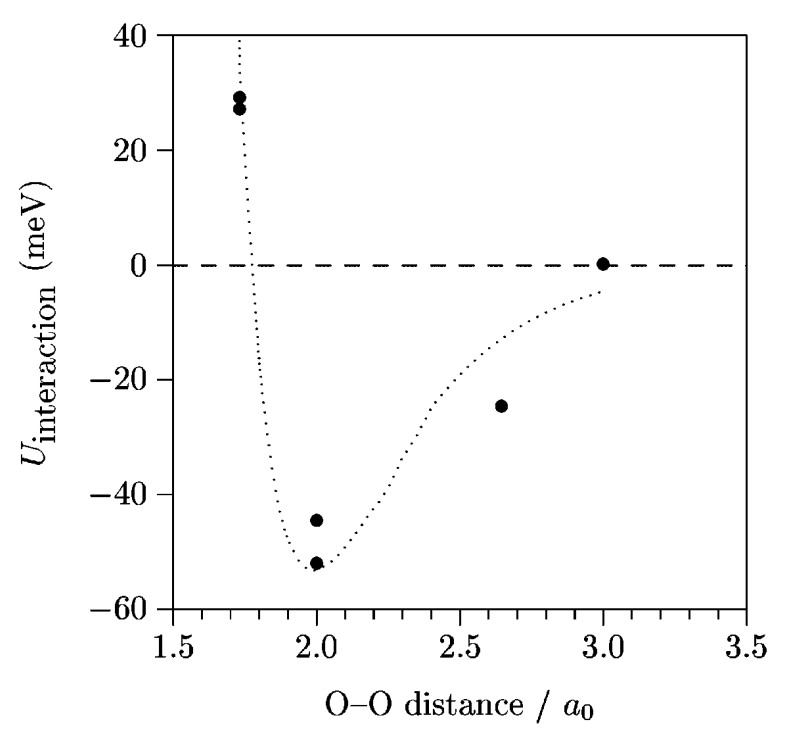

FIG. 5. Pair interaction potential, resulting from the modified dimer hopping rates.

interaction potential as plotted in Fig. 5. The two data points at $2 a_{0}$ and at $\sqrt{3} a_{0}$ result from the different ways by which these configurations can be reached.

The interaction potential shows the expected shape with a repulsive and an attractive branch. The minimum at $2 a_{0}$ is in agreement with the known behavior of oxygen atoms on $\mathrm{Ru}(0001)$ : The first ordered phase is a $(2 \times 2)$ structure [16], which forms islands indicating attractive interactions at $2 a_{0}$ [8]. The energy minimum of $-48 \mathrm{meV}$, still of the order of thermal energies, is consistent with strong thermal fluctuations observed for the $(2 \times 2)$ islands [8]. The energy difference between the $\sqrt{3} a_{0}$ and the $2 a_{0}$ sites $(80 \mathrm{meV})$ is in good agreement with the value of $70 \mathrm{meV}$ derived by Piercy et al. [17] from a combined LEED/Monte Carlo study of the melting of the $(2 \times 2)$ structure at $\Theta=0.25$. However, Fig. 4 demonstrates the operation of interactions beyond distances of $2 a_{0}$.

Although the shape of the potential is in the range of expectations for a chemisorbed, nonmetallic adsorbate such as oxygen, the data for the residence times demonstrate that also the dynamic behavior is significantly influenced by the interaction-each oxygen atom affects the residence time of a neighboring atom on 36 sites around it (apart from blocking its own site). The residence time on the six nearest-neighbor sites is apparently so short that it could not be observed here. For particles between $\sqrt{3} a_{0}$ and $3 a_{0}$ apart from each other, these times differ by more than 1 order of magnitude. These conclusions result from analyses of the hopping dynamics and would not be accessible from the knowledge of the (thermodynamic) interaction potential. They demonstrate the need to incorporate these effects in any concept modeling the kinetics of surface reactions.

*To whom correspondence should be addressed. Email address: renisch@ fritz-haber-institut.mpg.de

[1] R. Gomer, Rep. Prog. Phys. 53, 917 (1990).

[2] T. T. Tsong, Rep. Prog. Phys. 51, 759 (1988).

[3] G. Ehrlich and K. Stolt, Annu. Rev. Phys. Chem. 31, 603 (1980); G. Ehrlich, Appl. Phys. A 55, 403 (1992).

[4] T. Tsong, Phys. Rev. Lett. 31, 1207 (1973).

[5] T. R. Linderoth, S. Horch, E. Lægsgaard, I. Stensgard, and F. Besenbacher, Phys. Rev. Lett. 78, 4978 (1997).

[6] T. R. Linderoth, S. Horch, E. Lægsgaard, I. Stensgard, and F. Besenbacher, Surf. Sci. 402-404, 308 (1998).

[7] J. V. Barth, T. Zambelli, J. Wintterlin, R. Schuster, and G. Ertl, Phys. Rev. B 55, 12902 (1997).

[8] J. Wintterlin, J. Trost, S. Renisch, R. Schuster, T. Zambelli, and G. Ertl, Surf. Sci. 394, 159 (1996).

[9] S. Renisch et al. (to be published).

[10] J. D. Wrigley, M. E. Twigg, and G. Ehrlich, J. Chem. Phys. 93, 2885 (1990).

[11] J. Honerkamp, Stochastische Dynamische Systeme (VCH, Weinheim, 1990).

[12] C. Stampfl, S. Schwegmann, H. Over, M. Scheffler, and G. Ertl, Phys. Rev. Lett. 77, 3371 (1996).

[13] B. G. Briner, M. Doering, H.-P. Rust, and A. M. Bradshaw, Science 278, 257 (1997).

[14] C. Ratsch and M. Scheffler, Phys. Rev. B 58, 13163 (1998).

[15] This relation between the energy difference of equilibrium sites of the potential and the hopping rates does not change if we take into account that an intermediate state is involved; indeed, it is expected that the actual hopping process between two hcp sites proceeds via an intermediate fcc site.

[16] T. E. Madey, H. A. Engelhardt, and D. Menzel, Surf. Sci. 48, 304 (1975).

[17] P. Piercy, K. DeBell, and H. Pfnür, Phys. Rev. B 45, 1869 (1992). 